\title{
ArcheoSciences
}

Revue d'archéométrie

\section{Combustible pour la fabrication des cloches au Moyen Âge : l'exemple de la cathédrale Sainte- Marie de Nice (Alpes-Maritimes, France)}

Fuel for bell manufacturing in the Middle Ages: The example of Sainte-Marie cathedral in Nice (Alpes Maritimes, France)

Isabelle Gillot, Lise Damotte, Marc Bouiron et Claire Delhon

\section{OpenEdition}

Journals

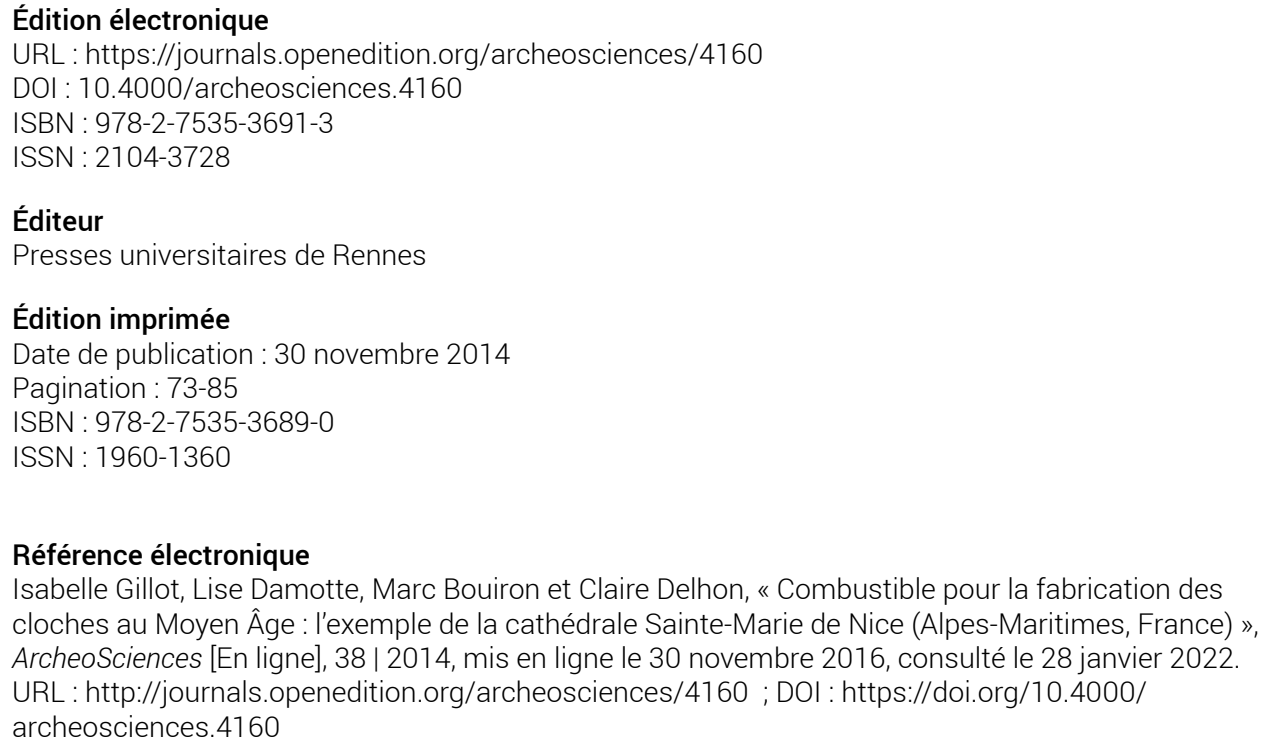




\title{
Combustible pour la fabrication des cloches au Moyen Âge : l'exemple de la cathédrale Sainte-Marie de Nice (Alpes-Maritimes, France)
}

\author{
Fuel for Bell Manufacturing in the Middle Ages: \\ The Example of Sainte-Marie Cathedral in Nice (Alpes Maritimes, France)
}

Isabelle Gillot ${ }^{a}$, Lise DAmotte ${ }^{\mathrm{b}}$, Marc Bouiron ${ }^{\mathrm{b}}$ et Claire DelhoN ${ }^{a}$

Résumé : Au Moyen Âge, les cloches étaient le plus souvent fondues dans des moules en terre placés et cuits dans des fosses creusées dans le sol à l'intérieur des églises ou dans un périmètre proche.

Si les vestiges des moules sont en général étudiés afin de déterminer la taille de la cloche et ses éventuelles inscriptions ou décors, il est rare que le contenu anthracologique des fosses soit étudié.

Grâce au Programme Collectif de Recherche en cours sur le site de «La colline du Château " à Nice (France), nous avons pu recueillir les fragments de charbons de bois associés aux fosses de coulée de cloche découvertes dans la cathédrale médiévale et procéder à une analyse antracologique. Les résultats obtenus sur deux fosses nous renseignent sur le contexte environnemental du site, sur l'approvisionnement en combustible pour la fabrication des cloches ainsi que sur les gestes techniques liés à la coulée des cloches médiévales.

L'essence représentée majoritairement est l'arbousier, associée principalement au chêne à feuillage caduc, au peuplier/saule et au chêne sclérophylle. Toutefois, on observe une grande biodiversité, qui renvoie à l'exploitation d'un ensemble de formations végétales, essentiellement méditerranéennes dont les plus représentées sont la chênaie mixte et la ripisylve.

\begin{abstract}
In the Middle Ages, bells were usually cast in clay moulds fired in pits excavated inside churches or nearby.
Fragments of such moulds are often studied to determine the size of the bells and possible inscriptions or decorations, but only rarely these pits have been examined for the presence of anthracological material (charcoal).

Thanks to the Collective Research Programme underway on the site "la colline du Chatteau " overlooking the city in Nice (Southern France), we were able to collect fragments of charcoal from two bell pits found in the nave of the mediaeval cathedral.

The results of anthracological analysis provided information on the environmental context, the origin of the wood used for making bells and technical aspects of casting mediaeval bells.

Most of the charcoal remains were from strawberry tree (arbutus), in association with deciduous oak, poplar/willow and sclerophyllous oak. A very high biodiversity was found, however, suggesting the exploitation of a wide range of plant formations, mainly Mediterranean, represented by a mixed-oak woodland and the riparian forests.
\end{abstract}

Mots clés : Anthracologie, Moyen Âge, Fabrication des cloches, environnement, combustible

Keywords: Charcoal analysis, Middle Ages, bell manufacturing, environment, firewood

\footnotetext{
a Université Nice-Sophia Antipolis, CEPAM - UMR 7264CNRS, Saint-Jean-d'Angély, 24, avenue des Diables-Bleus, 06357 NICE Cedex 4. (gillot@ unice.fr) (claire.delhon@cepam.cnrs.fr)

b Service Archéologie de la Ville de Nice, 16, rue François-Guisol, 06300 NICE. (lise.damotte@ville-nice.fr) (marc.bouiron@ville-nice.fr)
} 


\section{INTRODUCTION}

Au Moyen Âge, les cloches étaient le plus souvent fondues à l'intérieur des églises ou dans un périmètre proche. Les premières traces de ces opérations de fonte ont été découvertes il y a déjà quarante ans au Monastier de Vagnas en Ardèche (Thévenon, 1971). Toutefois, ces découvertes sont encore insuffisamment exploitées. Seuls les vestiges des moules sont en général prélevés et étudiés afin de déterminer la taille de la cloche et ses éventuelles inscriptions ou décors (Bernazzani, 2009; Gonon, 2002; 2010; Thomas 2009a). Cependant, les moules à cloches et leurs installations annexes peuvent fournir de précieuses indications sur l'évolution chronologique d'un site, la réfection ou la modification des églises (Thomas, 2009a).

Il est encore plus étonnant de constater que les fragments de charbon associés n'ont pas été systématiquement recueillis et encore moins analysés. À notre connaissance, les analyses de charbons provenant des fours et des fosses de moule à cloche sont encore très rares en France. Seules les études anthracologiques de deux ateliers de fondeurs, de cloches et de divers objets, (atelier dans la Villeneuve du Temple à Paris, Thomas 2009b, et site de l'Hôtel-Dieu à Marseille, Figueiral-Rowe, 2011 in Mellinand et Thomas, 2011) ont été rapportées jusqu’à présent. Cela conduit à retirer seule l'étude anthracologique d'Escaudes (Gironde, Bats, 2010) si cela est possible. Ces études pourraient apporter des informations sur les gestes techniques liés à la coulée des cloches médiévales, sur les questions économiques liées à l'approvisionnement en combustible (bois et charbon de bois) pour la fabrication des cloches et très certainement sur le contexte environnemental du site ou des sites en relation.

Un Programme Collectif de Recherche, dirigé par Marc Bouiron (Service Archéologie de la ville de Nice/CEPAM) se développe depuis 2006 sur le site de la colline du Château à Nice (Alpes Maritimes, France). La colline du Château est un grand rocher calcaire culminant à $93 \mathrm{~m}$ d'altitude, enserré dans l'actuelle ville de Nice (fig. 1), bordé par la mer Méditerranée en façade sud, la vallée du fleuve Paillon à l'ouest et par l'actuel port Lympia qui remplace depuis le XVIII ${ }^{e}$ siècle l'ancien vallon marécageux du même nom à l'est. Ces vallées sont abritées par un amphithéâtre de collines qui avoisinent les 300-350 m d'altitude à l'est et les $200 \mathrm{~m}$ d'altitude à l'ouest, dominées au nord par un mont plus élevé (le mont Chauve, $845 \mathrm{~m}$ d'altitude). Nice se situe à l'interface entre les étages bioclimatiques thermoméditerranéen et mésoméditerranéen (Ozenda, 1956). Dans les AlpesMaritimes, on observe des paysages très contrastés selon la localisation, humides en fond de vallon et plus secs sur les versants au soleil. Hors des zones de montagne, la végétation

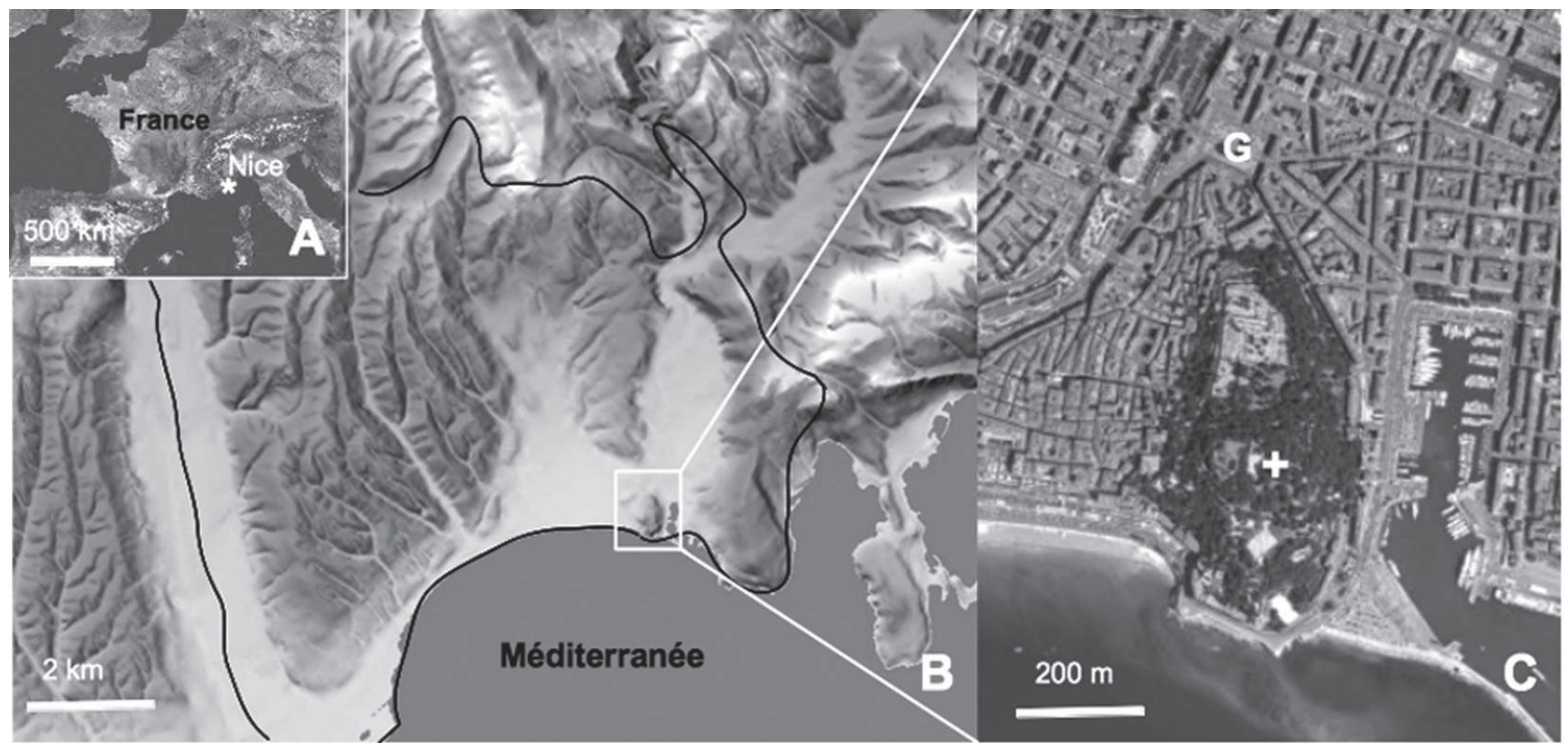

Figure 1 : Localisation de la ville de Nice et du site de la Colline du Château (images d'après http://www.geoportail.fr). A) Vue de la France avec la localisation de Nice (étoile). B) Limites approximatives de l'agglomération de Nice avec son relief. C) La colline du Château, avec l'emplacement de la cathédrale Sainte Marie (+) et la Place Garibaldi (G).

Figure 1: Localisation of the city of Nice and the site "La colline du Chatteau" (images from http://www.geoportail.fr). A) Nice is identified by an asterisk on the image of France. B) Nice and its topography. C) The Sainte Marie cathedral (+) and Garibaldi Square (G). 
est largement dominée par une chênaie (chêne à feuillage caduc, Quercus pubescens et chêne sclérophylle, Quercus ilex) caractéristique des étages mésoméditerranéen à supraméditerranéen d'adret. La zone d'étude, située en milieu urbain, reste néanmoins caractérisée par une végétation thermophile de la série du chêne vert. Le parc de la colline du Château est actuellement arboré avec des espèces indigènes majoritaires.

La colline abrite un important site archéologique en cours d'étude pour ses fortifications et son groupe cathédral. La cathédrale elle-même a fait l'objet de prospections et de premières fouilles aux $\mathrm{XIX}^{e}$ et $\mathrm{XX}^{\mathrm{e}}$ siècles, avant la reprise récente des opérations archéologiques (Bouiron, 2013). Cet édifice érigé dès le $\mathrm{v}^{\mathrm{e}}$ siècle, puis remanié durant le haut Moyen Âge, fait place à une première cathédrale au $\mathrm{XI}^{\mathrm{e}}$ siècle, ellemême reconstruite à la fin du Moyen Âge. Elle est détruite en 1706, lors du démantèlement de la fortification sur ordre de Louis XIV. Trois structures, une située dans le bas-côté sud et deux dans la nef centrale, témoignent de la fabrication de cloches dans l'emprise de la cathédrale (Damotte, 2011). Dans les trois cas, seules les structures « en creux " des installations, c'est-à-dire les fosses de coulée, sont conservées. Aucune trace des fours, construits en surface, n'a été repérée, car ils étaient en général détruits après la coulée (Gonon, 2000; Thomas, 2009b). Les fragments de charbons de bois issus des deux fosses de coulée des cloches de la nef centrale ont été recueillis afin de procéder à une analyse anthracologique.

Â travers cette thématique centrée sur les fosses de moule à cloches, nous avons tenté d'approcher deux problématiques : mieux comprendre une activité artisanale grâce à une de ses caractéristiques techniques et initier une approche écologique d'une région peu étudiée pour la période médiévale.

\section{Matériel et méthode}

\section{Matériel}

Le matériel étudié a été prélevé dans deux des trois fosses identifiées au sein de la cathédrale (fig. 2). Les deux fosses de moule à cloche, FS 321 et FS 388, ont donné lieu respectivement à quatre et à trois prélèvements correspondants à chaque unité stratigraphique (US) identifiée dans les remplissages (US 2080, US 2187, US 2186, US 2206 et US 2269, US 2291, US 2297; fig. 3 et 4). La fosse FS 240, située dans le bas-côté sud, avait été repérée et fouillée par F. Benoit en 1963 (Bouiron et Mercurin, 2007), qui l'avait interprétée comme un four de bronzier d'époque antique. Le dégagement de la structure en 2009 a permis de l'identifier comme une fosse destinée à recevoir un moule à cloche
(Damotte, 2011). Toutefois, cette structure, vidée lors des fouilles anciennes, n'a pas laissé de trace de comblement et nous ne l'avons donc pas incluse dans notre étude.

Les sédiments ont été prélevés et tamisés sur place à une maille de $4 \mathrm{~mm}$ et un jet d'eau assez fort. Seules les US 2206 et 2187, ont été tamisées au laboratoire à une maille de $2 \mathrm{~mm}$ et sous un jet d'eau modéré, puis triés sous la loupe binoculaire pour isoler les charbons de bois. La richesse en charbon de bois s'est avérée très variable d'un prélèvement à l'autre : le tamisage de cinq litres de sédiment de l'US 2187 a permis d'isoler 24 fragments alors qu'un litre de l'US 2206 a fourni 225 fragments.

Les sols associés aux fosses de coulée de cloches n'étant pas conservés, les charbons provenant d'une tranchée de fondation et de trous de poteaux proches des fosses, peutêtre liés à l'installation d'échafaudages pour le chantier de la cathédrale, ont également été analysés à titre de comparaison (US 2104, US 2161, US 2165, US 2182, US 2180).

\section{Analyse anthracologique}

Après fracturation à la main afin d'obtenir des coupes fraîches des trois plans anatomiques du bois, chaque fragment est observé au microscope photonique à réflexion fond clair/fond noir (Leica DMR) à des grossissements de x 100 à $\mathrm{x} 500$. Les caractères anatomiques observés sont comparés aux critères de détermination publiés dans les atlas d'anatomie du bois (Schweingruber, 1978; Vernet et al., 2001) et avec ceux des charbons actuels de la collection de référence du laboratoire (CEPAM, UMR 7264). Selon les cas, la détermination atteint le niveau de la famille ou sous-famille (Rosaceae Maloideae : les maloïdées), du genre (Ulmus sp., l'orme), d'un groupe de genres (Populus sp./Salix sp., le peuplier ou le saule), d'un groupe d'espèces (Quercus f.c., les chênes à feuillage caduc) ou, rarement, de l'espèce (Ostrya carpinifolia, le charme-houblon).

Lorsque les US ont fourni un nombre réduit de fragments (moins de 200), la totalité du corpus est analysée. Pour l'US 2269, dont les charbons sont abondants, nous avons analysé un effectif de 297 fragments dont 277 ont pu être identifiés. La courbe effort-rendement de cette US présente un palier qui permet de penser que le spectre de la biodiversité de l'assemblage anthracologique est complet (Chabal, 1997).

L'abondance des différents taxons est exprimée en nombre de fragments. Les abondances relatives (en pourcentage) sont calculées sur le total des charbons identifiés (voir tableau 1). Les charbons indéterminés représentent $12,5 \%$ de la totalité des fragments étudiés. Pour la facilité de lecture, le taxon nommé Populus sp./Salix sp. regroupe les fragments identifiés comme Salix sp., Populus sp. et ceux 


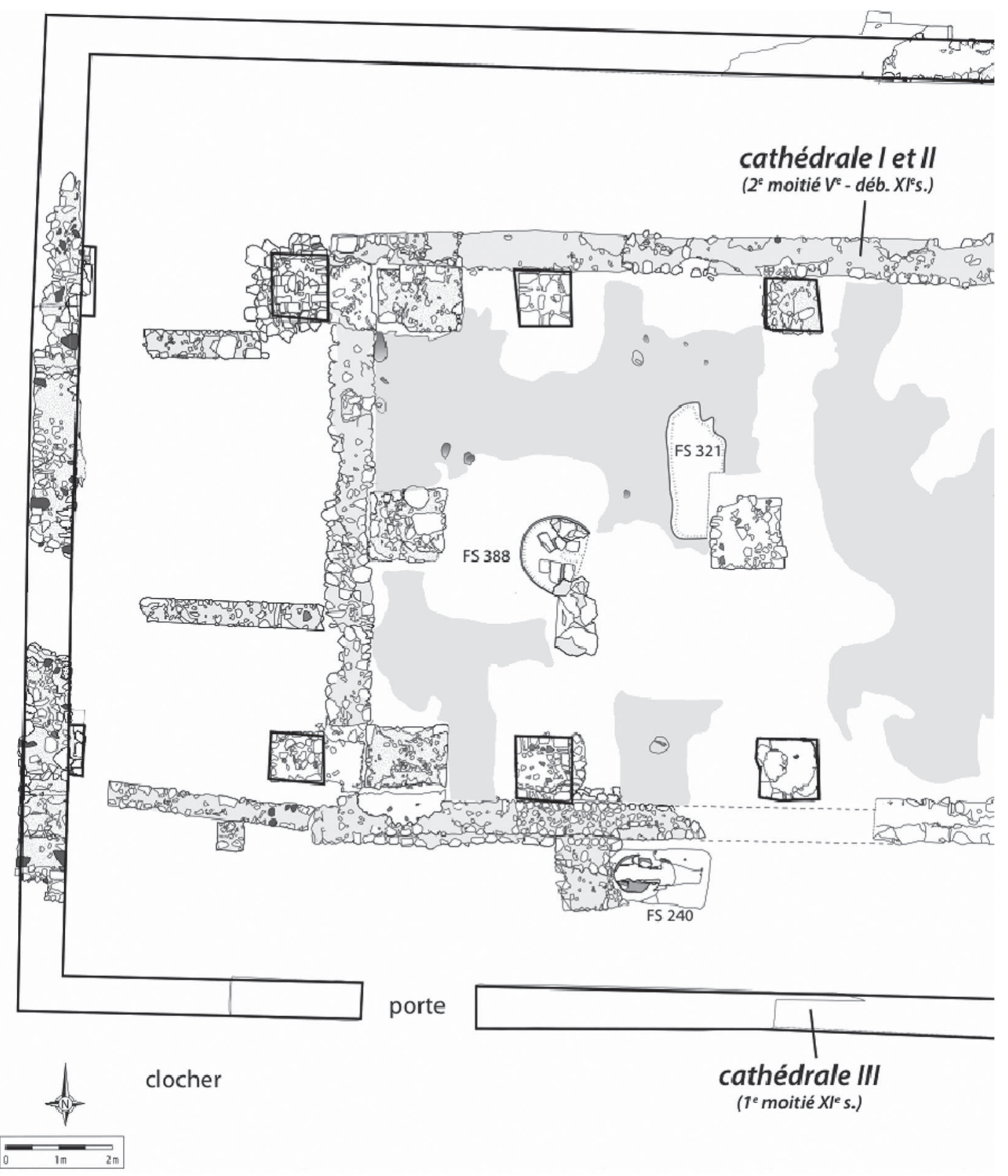

Figure 2 : Plan d'une partie de la fouille archéologique de la cathédrale Sainte Marie avec localisation des trois fosses de coulée de cloche FS 388, FS 321 et FS 240 (DAO M. Bouiron \& L. Damotte/Service Archéologie de la Ville de Nice).

Figure 2: Map of a part of the archaeological excavation of the Sainte-Marie Cathedral showing the three bell pits, FS 388, FS 321 and FS 240 (DAO M. Bouiron \& L. Damotte/SAVN). 

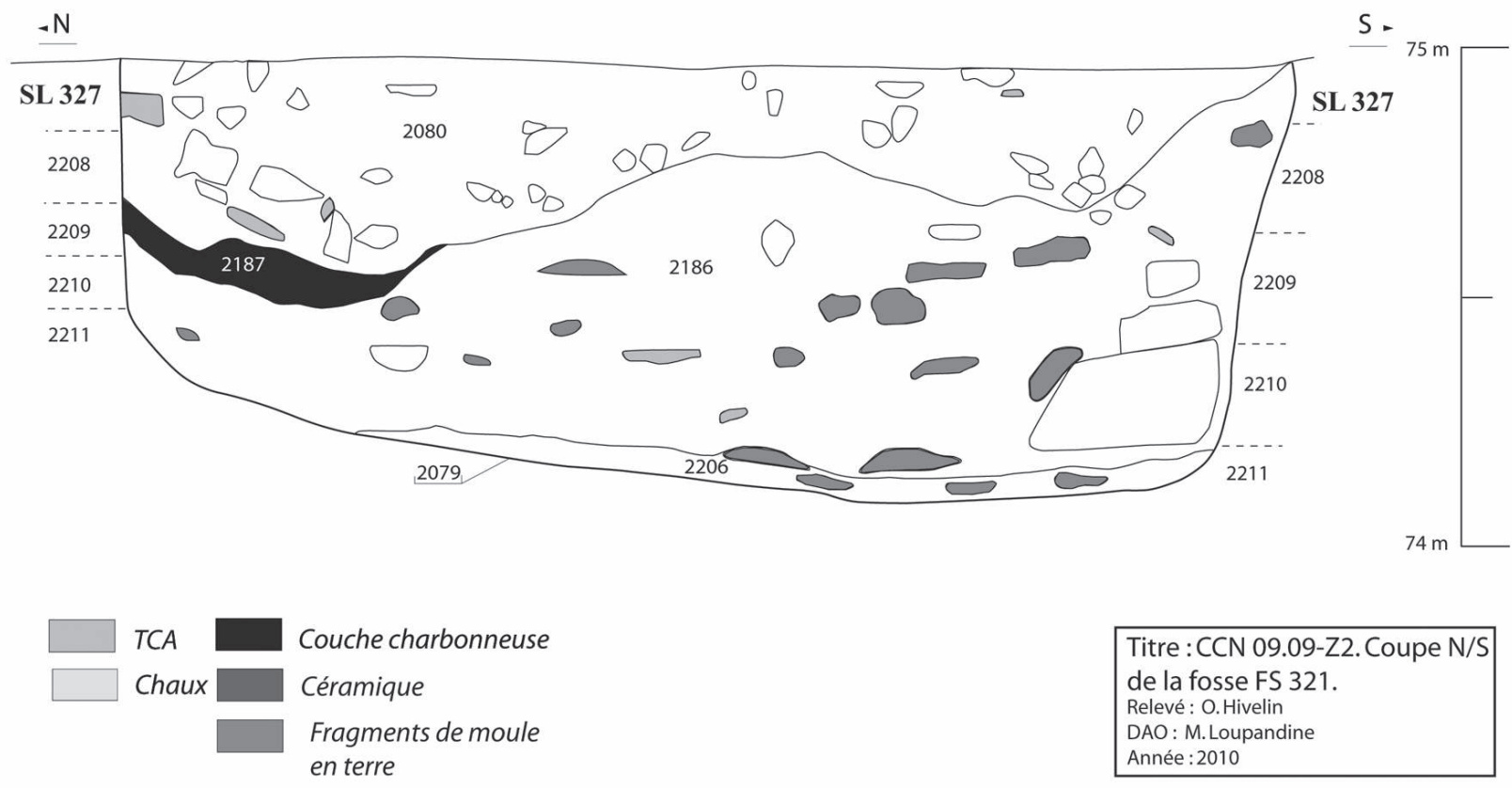

Figure 3 : Coupe nord/sud de la fosse FS 321.

Figure 3: North/south section of bell pit FS 321.

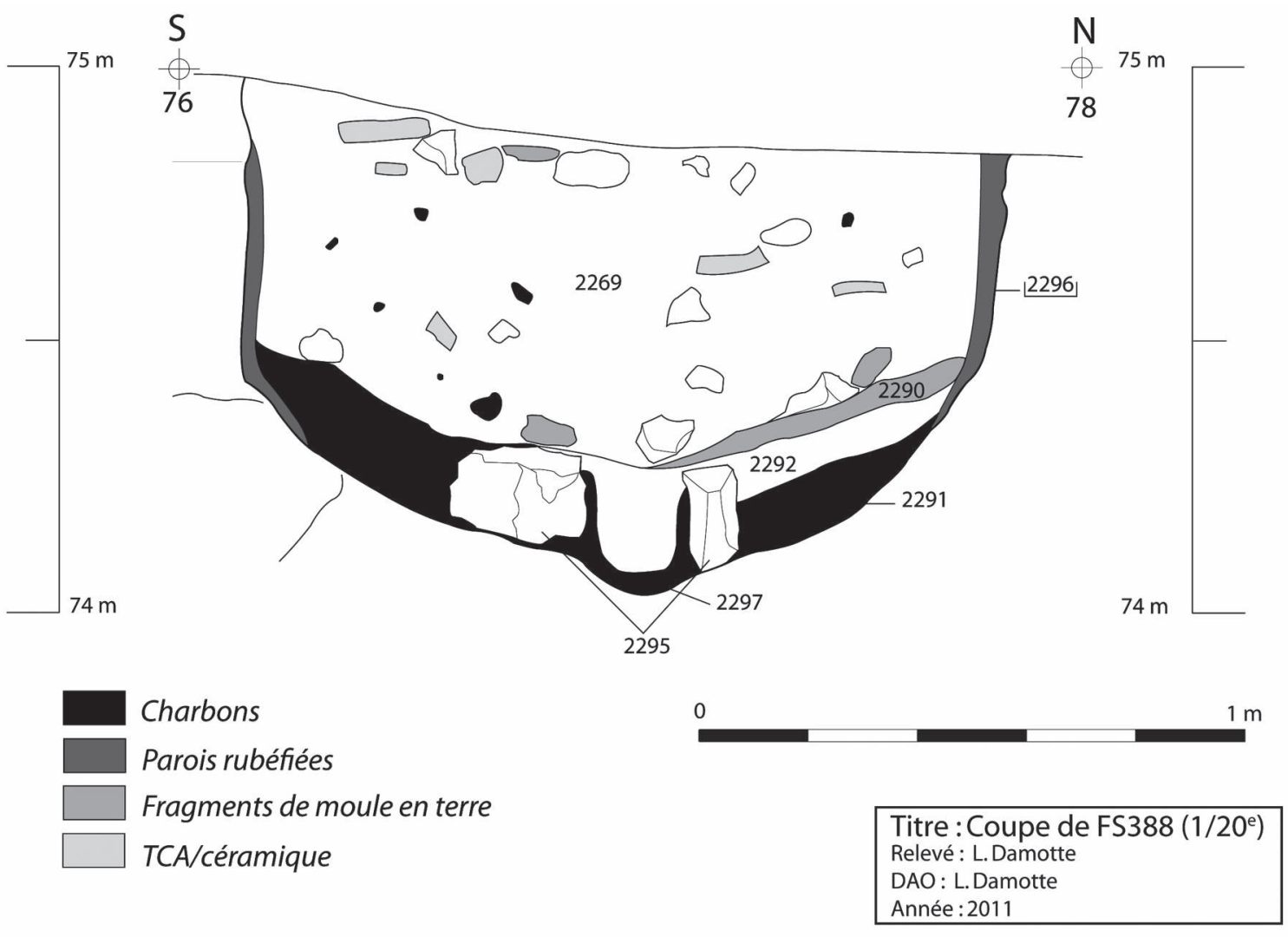

Figure 4 : Coupe sud/nord de la fosse FS 388.

Figure 4: South/north section of bell pit FS 388. 


\begin{tabular}{|c|c|c|c|c|c|c|c|c|c|c|c|c|c|c|c|c|c|c|c|c|c|c|c|c|c|c|c|c|}
\hline 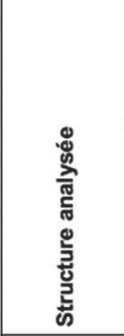 & 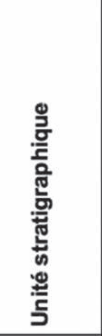 & 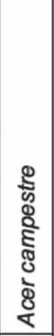 & $\begin{array}{l}0 \\
0 \\
0 \\
0 \\
\frac{5}{4}\end{array}$ & 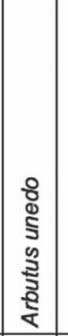 & 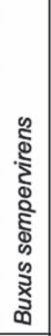 & $\begin{array}{l}0 \\
0 \\
0 \\
0 \\
5 \\
0 \\
0\end{array}$ & 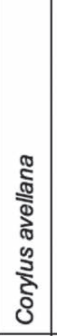 & 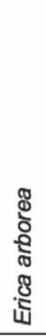 & 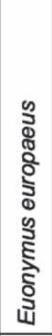 & 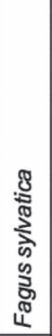 & 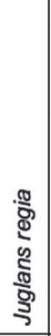 & 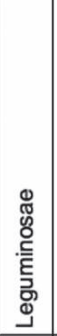 & 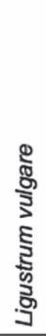 & $\begin{array}{l}\mathbb{\Xi} \\
\mathbb{\delta} \\
\frac{0}{0} \\
\frac{0}{\pi} \\
\Sigma\end{array}$ & 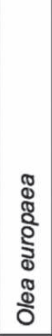 & 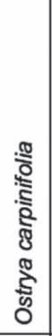 & 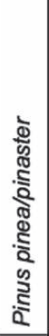 & 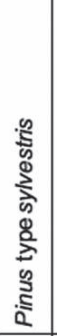 & 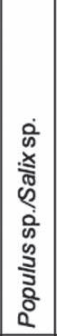 & 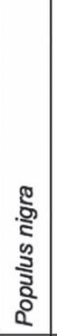 & 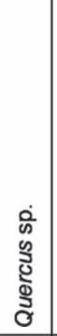 & 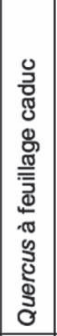 & 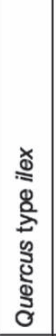 & 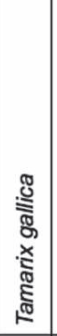 & $\begin{array}{l}\text { Dे } \\
\text { के } \\
\text { है } \\
\text { है }\end{array}$ & $\begin{array}{l}\text { S } \\
\text { S } \\
\text { E } \\
\text { है } \\
\text { ș }\end{array}$ & 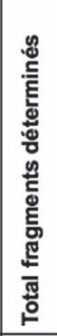 & 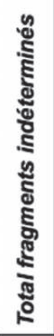 \\
\hline \multirow{4}{*}{ Fosse 321} & 2080 & & & & & & & 2 & & & & & & & 1 & 2 & & 2 & & & & 2 & & & 3 & & 12 & 3 \\
\hline & 2187 & & 3 & 2 & 1 & & & & & & & & & & 1 & & 1 & & 72 & & 4 & 11 & 1 & & 6 & & 102 & 32 \\
\hline & 2186 & 1 & & 6 & & 7 & & 3 & 1 & & 1 & & & 1 & & 1 & 1 & 1 & 15 & 2 & 2 & 13 & 6 & 1 & 16 & & 78 & 15 \\
\hline & 2206 & & 1 & 3 & & & 1 & & & & & 2 & & 1 & & & & & 84 & 1 & & 6 & & & & & 99 & 32 \\
\hline \multirow{3}{*}{ Fosse 388} & 2269 & & 8 & 122 & & & & 8 & & & & & 1 & 3 & 11 & 2 & 2 & & 7 & 1 & 4 & 70 & 37 & & & 1 & 277 & 20 \\
\hline & 2291 & & & 67 & & & & & & & & & & & & & & 1 & & & & 43 & 10 & & & & 121 & 0 \\
\hline & 2297 & & 1 & 41 & & & & & & & & & & & & & & & & & 2 & 51 & 48 & & & & 143 & 3 \\
\hline \multirow{5}{*}{$\begin{array}{l}\text { Trous de } \\
\text { poteaux }\end{array}$} & 2104 & & 2 & 8 & & & & 2 & & & & & 1 & 1 & 1 & 2 & 2 & 2 & 1 & & 1 & 4 & 12 & & 1 & & 40 & 16 \\
\hline & 2161 & & & 1 & & & & 3 & & & & & & & & & & & & & & & 1 & & & & 5 & 1 \\
\hline & 2165 & & & & & & & 1 & & & & & & & 1 & & & & & & & & 2 & & & & 4 & 1 \\
\hline & 2182 & & 1 & 1 & & & & & & & & & & & & & & & & & & & & & & & 2 & 1 \\
\hline & 2180 & & & 2 & & & & 1 & & 1 & & & & & & 1 & 1 & & & & & & & & 2 & & 8 & 3 \\
\hline \multicolumn{2}{|c|}{ Total fragments/taxons } & 1 & 16 & 253 & 1 & 7 & 1 & 20 & 1 & 1 & 1 & $\underline{2}$ & 2 & 6 & 15 & 8 & 7 & 6 & 179 & 4 & 13 & 200 & 117 & 1 & 28 & 1 & 891 & 127 \\
\hline \multicolumn{2}{|c|}{ Pourcentage } & 0,1 & 1,8 & $|28,4|$ & 0,1 & 0,8 & 0,1 & 2,2 & 0,1 & 0,1 & 0,1 & 0,2 & 0,2 & 0,7 & 1,7 & 0,9 & 0,8 & 0,7 & 20,1 & 0,4 & 1,5 & 22,4 & 13,1 & 0,1 & 3,1 & 0,1 & 100 & 12,5 \\
\hline
\end{tabular}

Tableau 1 : Résultats de l'analyse anthracologique des fosses FS 321 et FS 388 et des cinq trous de poteaux.

Table 1: Result of charcoal analysis from pits FS 321 and FS 388 and the five postholes.

pour lesquels il n'a pas été possible de trancher entre Salix et Populus qui sont très proches anatomiquement. (Vernet et al., 2001). Seul Populus nigra peut parfois être identifié à l'espèce par ses rayons bisériés (Vernet et al., 2001). Bien qu'il soit impossible de différencier les espèces de chêne par le biais de l'anatomie comparée, le taxon Quercus à feuillage caduc est probablement Quercus pubescens (Chêne blanc ou pubescent), étant donné la localisation du site et la végétation actuelle. De même, il semble raisonnable de proposer que le chêne sclérophylle présent dans nos assemblages soit principalement le chêne vert (Quercus ilex), d'autant plus qu'il est peu exigeant quant à la nature chimique du substrat (Quezel, 1976) sans exclure la présence de chêne kermès (Quercus coccifera).

Les résultats sont présentés sous forme de spectres de fréquences (\%) ordonnés selon la stratigraphie en diagramme anthracologique.

\section{Datation}

Les fosses ont fait l'objet de datations radiocarbone par AMS (société Amélie, Études environnementales \& archéologiques) sur des charbons préalablement identifiés et sélectionnés pour leur association avec le fonctionnement du four de coulée de cloche. Des espèces à courte durée de vie et à croissance rapide ont été choisies afin d'éviter les effets "vieux bois".

ArCheoSCIEnCes, revue d'archéométrie, 38, 2014, p. 73-85

\section{Résultats}

Au total, 1018 fragments de charbons de bois provenant de douze unités stratigraphiques ont été observés. Huit cent quatre-vingt onze d'entre eux ont pu être identifiés avec une précision variable. Ces charbons ont une taille comprise entre $2 \mathrm{~mm}$ et quelques centimètres de longueur environ, avec une proportion de fragments millimétriques majoritaires et une buchette de plusieurs centimètres dans l'US 2297. Les fragments sont dans un bon état de conservation. Les caractères anatomiques sont, en général, bien conservés. Les fentes radiales de retrait sont rares et le taux de vitrification est très faible.

Lanalyse anatomique de 891 charbons de bois a permis d'identifier 23 taxons différents représentatifs (tab. 1). On note la présence de gymnospermes (pins : pin pignon/pin maritime, Pinus pinealpinaster et pin du type sylvestre, Pinus type sylvestris) et d'angiospermes arborescentes (arbousier, Arbutus unedo; aulne, Alnus sp.; charme-houblon, Ostrya carpinifolia; chênes, Quercus sp. dont le chêne à feuillage caduc, Quercus f.c. et le chêne sempervirent, Quercus type ilex; érable champêtre, Acer campestre; hêtre, Fagus sylvatica; maloïdées, Maloideae; noyer, Juglans regia; orme, Ulmus sp.; olivier, Olea europaea; peuplier/saule, Populus sp./Salix sp.; troène, Ligustrum vulgare) et arbustives (grande bruyère, Erica arborea; buis, Buxus sempervirens, cornouiller, Cornus 
sp.; fusain, Euonymus europaeus ; noisetier, Corylus avellana ; tamaris, Tamaris gallica; viorne, Viburnum tinus).

Toutes structures confondues, l'arbousier, avec $28,4 \%$, est de loin l'essence la plus représentée, suivi par le chêne à feuillage caduc $(22,4 \%)$, puis l'ensemble peuplier/saule qui représente $20,1 \%$ de la totalité des fragments, et enfin le chêne type ilex $(13,1 \%)$. L'orme et la grande bruyère représentent respectivement $3,1 \%$ et $2,2 \%$ des fragments alors que les autres taxons sont plus faiblement représentés avec des fréquences relatives inférieures à $2 \%$ (fig. 5).

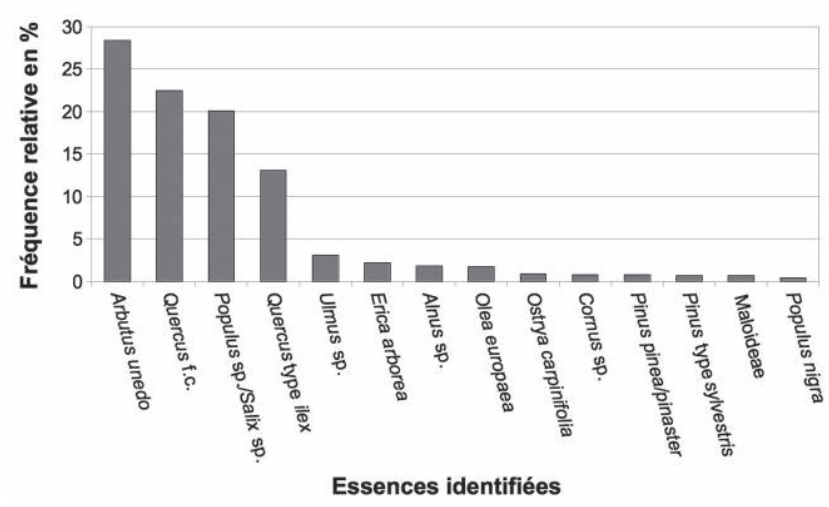

Figure 5 : Fréquence relative des principaux taxons dans l'ensemble des structures. Les taxons présentant une fréquence relative inférieure à $0,4 \%$ ne sont pas représentés (Acer campestre, Buxus sempervirens, Corylus avellana, Euonymus europaeus, Fagus sylvatica, Juglans regia, Ligustrum vulgare, Leguminosae, Viburnum tinus, Tamarix gallica).

Figure 5: Relative frequency of the main taxa in all structures. Taxa with a frequency $<0.4 \%$ are not shown (Acer campestre, Buxus sempervirens, Corylus avellana, Euonymus europaeus, Fagus sylvatica, Juglans regia, Ligustrum vulgare, Leguminosae, Viburnum tinus, Tamarix gallica).

\section{Fosse FS 388}

Treize taxons sont représentés dans cette fosse dont les trois plus abondants sont l'arbousier, le chêne à feuillage caduc et le chêne vert, qui totalisent respectivement $42,5 \%$, $30,3 \%$ et $17,6 \%$ des fragments identifiés (fig. $6 \mathrm{~A}$ ). Avec un total de 12 essences, l'US 2269, comblement supérieur de la fosse FS 388, est plus diversifiée que les US 2291 et US 2297 (fig. 6A), qui ne totalisent que 4 essences : l'arbousier, le chêne à feuillage caduc et le chêne vert majoritairement, avec seulement deux fragments de pin type sylvestre et d'aulne respectivement (fig. 6A). La buchette retrouvée en place au fond de la fosse FS 388 est un morceau de chêne à feuillage caduc.
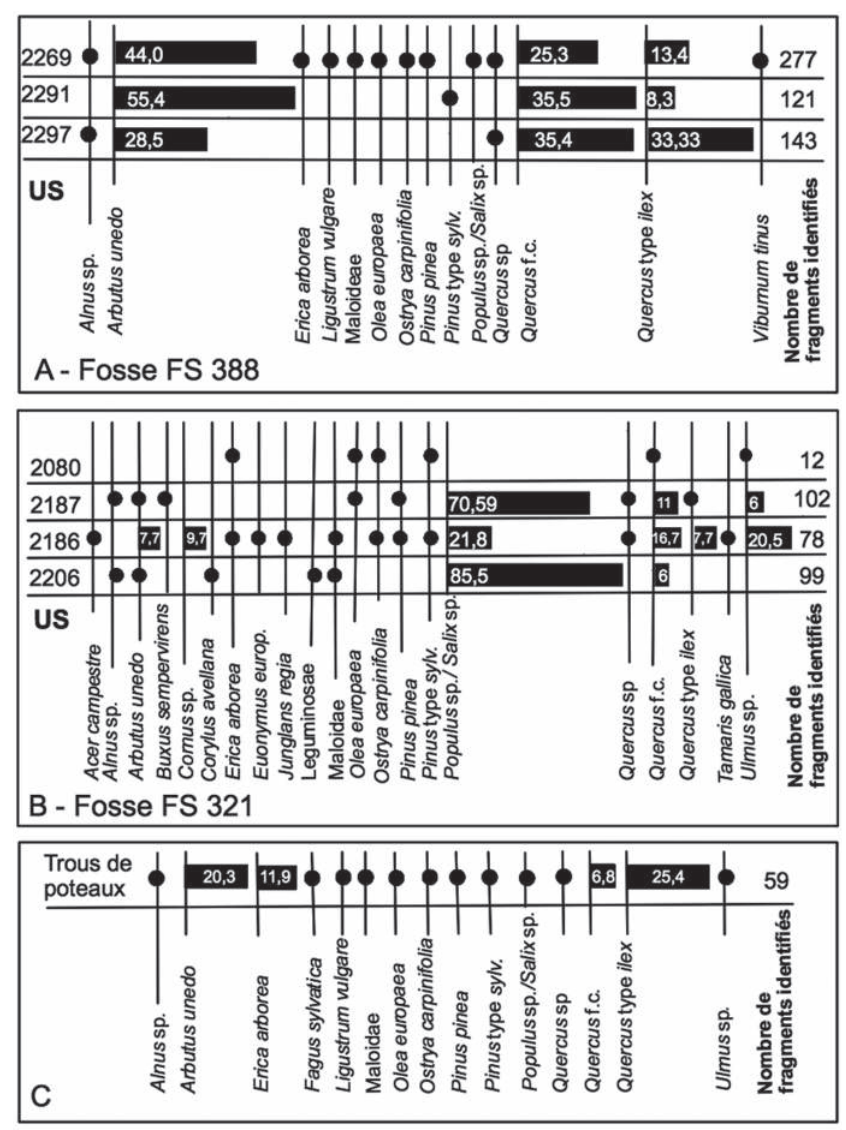

Figure 6 : Diagramme anthracologique des différentes structures. A) Les trois US de la fosse FS 388; B) Les quatre US de la fosse FS 321 ; C) Ensemble des charbons des cinq trous de poteaux contemporains des fosses FS 388 et FS 321. Les chiffres en blanc indiquent le pourcentage de chaque taxon. Cercle plein : pourcentage de taxon inférieur à $3 \%$ (A) ou nombre de fragments compris entre 1 et 3 (B et C). Quercus f.c., Quercus à feuillage caduc.

Figure 6: Charcoal analysis diagram of the different structures. A) The three stratigraphic units of the pit FS 388; B) The four stratigraphic units of pit FS 321. C) All charcoal from the five postholes. The numbers in white indicate the percentage of taxa. Dark circles: percentage of taxa $<3 \%$ ( $A$ ) or number of charcoal between 1 to 3 (B and $C$ ). Quercus f.c., deciduous Quercus.

\section{Fosse FS 321}

Toutes les US sont assez diversifiées : l'US 2186, la plus hétérogène, contient quinze essences différentes alors que l'US 2080, la moins variable, au sommet de la fosse FS 321, contient tout de même six essences différentes (fig. 7). Ainsi, les quatre US de la fosse FS 321 présentent un ensemble de vingt taxons (fig. 6B) dont neuf taxons ne sont pas présents dans la fosse FS 388. Il s'agit du buis, du cornouiller, de l'érable, du fusain, d'une légumineuse, du noisetier, du noyer, de l'orme et du tamaris. En revanche, le troène et 


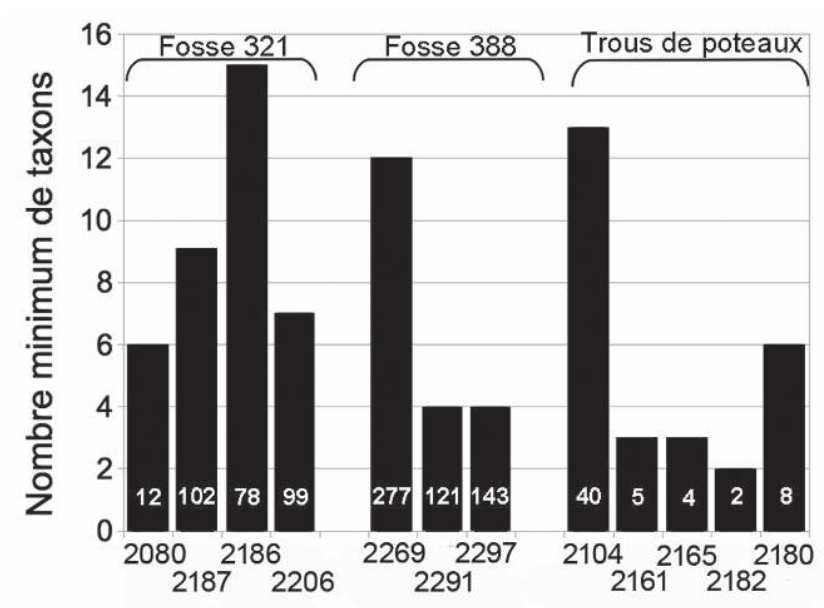

Unités Stratigraphiques

Figure 7 : Biodiversité (en nombre minimum de taxons par unité stratigraphique, US) dans les fosses FS 321 et FS 388 et les cinq trous de poteaux. En blanc: nombre de fragments de charbons de bois identifiés. Pour les deux fosses, les résultats sont présentés des US superficielles vers les US profondes de gauche à droite.

Figure 7: Biodiversity (minimum number of taxa for each Stratigraphic Unit (US) in pits FS 321 and FS 388 and in the five postholes. The number in white indicate the charcoal fragments identified. For pits FS 321 and FS 388, the results are presented from the top levels down to the deepest levels from left to right.

la viorne-tin qui étaient présent dans la fosse FS 388 sont absents dans la fosse FS 321. Le taxon peuplier/saule présente un pourcentage très élevé $(58,8 \%)$ dans la fosse FS 321, et sa concentration décroît depuis l'US du fond de fosse jusqu'à être totalement nulle au sommet. Le chêne à feuillage caduc $11 \%$ et l'orme 8,6\% sont les deux taxons les plus représentés après le peuplier/saule.

\section{Trous de poteaux}

En dépit du faible effectif des assemblages, quatorze taxons, déjà rencontrés dans les fosses FS 388 et FS 321, sont présents (chêne, un représentant des Rosacées Maloïdées, troène, arbousier, bruyère arborescente, peuplier/saule, charme-houblon, orme, aulne, olivier et pin; fig. 6C) avec une nette dominance du chêne vert, de l'arbousier et de la bruyère arborescente, respectivement $25,4 \%, 20,3 \%$ et 11,9 \%. Le hêtre apparaît dans l'US 2180. Malgré le nombre réduit de fragments de charbon de bois dans ces structures, la diversité végétale reste plutôt élevée avec un nombre d'essences variant de deux à treize (fig. 7).

\section{Datation}

La fosse FS 321 (US 2206) a été datée par radiocarbone de $1050 \pm 30$ BP (Beta-334375) sur un fragment de peuplier (Populus sp.), soit Cal AD 898-1027 à 95,4 \% de probabilité. La fosse FS 388 (US 2297) a été datée de $1070 \pm 30$ BP (Beta-334376) sur un fragment d'aulne (Alnus sp.), soit Cal AD 895-1021 à 95,4 \% de probabilité. Le croisement des deux permet de placer soit au début du $\mathrm{x}^{\mathrm{e}}$ siècle, soit avec beaucoup plus de vraisemblance autour de l'an Mil, l'utilisation de ces deux fosses. En effet, elles ont été creusées dans le sol de la vieille cathédrale paléochrétienne, probablement au moment où celle-ci est en travaux pour la construction d'un nouvel édifice élargi (le sol n’a pas été reconstitué immédiatement après le creusement des fosses). Cette nouvelle cathédrale est consacrée en 1049.

\section{Discussion}

Bien que les structures étudiées soient différentes (deux fosses de coulée de cloche et un ensemble de trous de poteaux), elles forment un ensemble qui apparaît chronologiquement cohérent. En effet, d'après les datations radiocarbone, les deux fosses sont contemporaines, et les trous de poteaux sont attribués au chantier de construction de la même période. Il est possible que les rejets de charbons dans les remblais proviennent pour partie du four de fusion du métal, du four pour cuire les moules de fonderie ou les décirer, ou enfin même du combustible utilisé pour les autres activités artisanales et domestiques des ouvriers. Si la majeure partie des comblements supérieurs des fosses est composée des sédiments issus des strates de creusement et donc antérieures, comme cela est attesté par la présence de céramique datée de l'Antiquité tardive (Damotte, 2011), on peut légitimement proposer qu'une grande partie des charbons soient contemporains de la coulée de cloche grâce à la présence des débris de moule. Toutefois, nous n'excluons pas la possibilité de la présence de charbons résiduels non représentatifs de la période médiévale.

\section{Des charbons concentrés}

De façon générale, en anthracologie, les prélèvements focalisés sur des charbons concentrés dans des structures en creux (fosse, trou de poteau) sont suspectés d'une représentativité paléoenvironnementale non optimale (Chabal, 1997; Delhon, 2006) car ils correspondent souvent à un ramassage ponctuel, et donc à un échantillonnage non-exhaustif de la végétation. En revanche, ils peuvent apporter des informations précieuses sur les pratiques humaines. 
Nos résultats pour les trois structures de combustion montrent des différences sensibles. Seuls les individus du lit de charbons de bois encore en place dans le fond de la fosse FS 388 (US 2297 et 2291) semblent directement liés à l'utilisation de la fosse pour la fabrication de la cloche. En effet, comparé à la fosse FS 321 et aux comblements, le nombre de taxons dans ces deux US de la fosse FS 388 est très faible, avec trois essences majoritaires (arbousier, chêne à feuillage caduc et chêne vert) et une quatrième représentée par un unique fragment. Le lit de charbons des US 2297 et 2291 correspond en quelque sorte à un instantané lors de l'utilisation de la structure, tandis que le comblement US 2269 représente plutôt une phase d'abandon comme pour la fosse FS 321 et les trous de poteaux. De plus, ces unités stratigraphiques, très diversifiées en taxons, présentent aussi des tessons de céramique et quelques fragments de verre et surtout une quantité très importante de restes d'ossements (169) supposant l'utilisation de la structure comme fosse de rejet après son utilisation pour la fabrication de la cloche (Bouiron, 2011). Dans ces structures, les charbons en position secondaire proviennent probablement d'un ou plusieurs rejets et non d'un événement de combustion ponctuel. De ce fait, si l'on tient compte de l'ensemble des résultats, le spectre de végétation, s'il n'est pas complet, apparaît assez large pour être interprété.

Le spectre anthracologique présente une biodiversité totale relativement élevée ( 23 taxons). Pour comparaison, on trouve de 15 à 25 espèces par spectre à l'âge du Fer en Languedoc, zone de moins grande diversité que la notre, avec un maximum de 35 taxons par site (Chabal, 1994). La récolte et l'utilisation du bois ne semblent pas très sélectives, l'échantillonnage réalisé lors de la récolte du combustible semble conforme à la végétation-source et, en conséquence, ces données permettent de formuler de premières interprétations.

\section{Paléoenvironnement}

Les individus ligneux identifiés dans les fosses trouvent des homologues actuels dans différentes associations végétales (Ozenda, 1975), ou corroborent les travaux antérieurs. En effet, les seuls résultats de palynologie disponibles à proximité du site sont issus d'un sondage carotté profond à l'emplacement de la Place Garibaldi (G en fig. 1), sur le piémont nord de la colline du Château (Sivan et Court-Picon, 2007), et sont chronologiquement mal définis. Pour une période allant probablement de l'âge de Bronze à la fin du Moyen Âge, ils proposent une prairie humide régulièrement fauchée aux abords immédiats du Paillon, avec quelques saules, frênes et bouleaux en bordure du cours d'eau. Pour les auteurs, «Ces formations végétales sont accompagnées d'une végétation rudérale de milieux très fréquentés et labourés » et "le paysage végétal environnant doit être imaginé comme de vastes prairies pâturées au sein desquelles quelques parcelles sont réservées pour la culture des céréales " (Sivan et CourtPicon, 2007, p. 16). La vigne, l'olivier, le noyer et le châtaignier, ce dernier apparaissant plus tardivement, seraient présents dans la région, mais ne seraient pas cultivés à proximité du sondage palynologique. "Le chêne pubescent forme encore, çà et là, avec l'érable, l'orme et le tilleul, quelques bosquets d'importance modeste. Le sapin et le pin sylvestre composent les peuplements d'altitude des montagnes les plus proches " (Sivan et Court-Picon, 2007, p. 16). Nous retrouvons certaines de ces espèces dans notre analyse : les contextes humides, qui peuvent évoquer la ripisylve du Paillon, transparaissent dans l'US 2186, avec la présence de l'orme, du fusain, du cornouiller, du charme-houblon, du noyer de même que dans l'US 2206 avec la présence du peuplier noir, peut-être d'autres peupliers et/ou du saule et de l'aulne.

L'assemblage des taxons identifiés indique que le combustible provient de plusieurs milieux différents :

- une chênaie mixte méditerranéenne associée à des espèces arbustives ou buissonnantes, avec pour taxons caractéristiques le chêne à feuillage caduc, l'érable, le troène, le cornouiller et une maloïdée peut être de type Sorbus. Ce dernier est un taxon héliophile que l'on retrouvera plutôt en milieu ouvert, c'est-à-dire en lisière de forêt.

- une chênaie mixte dégradée dominée par des espèces sempervirentes, en particulier le chêne vert, et les espèces associées comme l'arbousier, la grande bruyère ou encore la viorne-tin et le cornouiller. Des légumineuses, que nous n'avons pas été en mesure d'identifier plus précisément, peuvent indiquer des milieux dégradés.

- une basse plaine avec des niveaux phréatiques assez élevés, caractérisée par des espèces mésophiles, forestières ou arbustives telles que l'orme et le fusain d'Europe, ainsi que des espèces littorales telles que le tamaris. Le cornouiller peut être aussi associé à ce milieu. Le charme-houblon peut être associé soit au milieu précédent, soit se trouver parmi les espèces hygrophiles riveraines ou de milieux très humides (ripisylves-marécages), telles que les saules et les peupliers, ou encore l'aulne.

Dans ce spectre floristique, se trouvent des espèces dont les fruits ou les graines peuvent être consommées, telles que l'olivier, le noyer, le pin pignon/maritime ou le sorbier. La culture de l'olivier est probable, celle du noyer est possible; néanmoins, toutes ces espèces peuvent aussi bien être sauvages, spontanées ou subspontanées. 
Enfin, le pin sylvestre, le buis, le hêtre ou le noisetier signalent une végétation des étages supraméditerranéen à montagnard. En effet, le noisetier, bien que rustique, ne pousse pas sur le littoral méditerranéen (Rameau et al., 2008).

Cette végétation correspond également à des sols différents et variés : le maquis, dont l'arbousier associé à la bruyère arborescente sont typiques, résulte de la dégradation de la forêt de chênes sclérophylles, elle-même ayant généralement remplacé un peuplement originel de chênes à feuillage caduc (Pons et Quezel, 1998) et s'établit plutôt sur des sols siliceux ou en terrain décarbonaté. Parallèlement, la garrigue, constituée du chêne vert, de l'olivier et de l'arbousier correspond à la série de dégradation de la chênaie sur terrain calcaire.

Il est possible que certaines espèces aient été choisies car considérées comme appropriées pour le feu alors que d'autres essences non représentées ne l'étaient pas. Mais on peut aussi envisager que la sélection du combustible s'est faite en fonction du calibre plutôt que de l'essence, la bruyère, l'arbousier et le cornouiller fournissant les petits calibres recherchés. On remarquera aussi l'absence du frêne parmi les taxons de ripisylve, qui fait écho à son absence signalée par Sivan et Court-Picon (2007) dans le spectre pollinique de la place Garibaldi sans qu'il nous soit possible d'en proposer une explication.

\section{Nature de l'approvisionnement}

L'analyse anthracologique montre la coexistence dans les spectres de plusieurs associations végétales dont les zones de répartition sont disjointes. Il est intéressant de noter la présence, faible mais réelle, de taxons collinéens à montagnards, tels que le pin sylvestre ou le hêtre, à proximité de l'étage thermoméditerranéen. En l'absence de sources écrites pouvant nous orienter, l'analyse des charbons de bois de ces fosses nous permet d'envisager la possibilité d'une récolte opportuniste aux environs immédiats de la fosse, avec des végétaux, collectés au pied de la colline, peut être associés à du bois de récupération (bois d'œuvre, outils). Le four de fusion n'ayant pas été retrouvé (Bouiron, 2011), il n'est pas possible de comparer les deux combustibles. On peut aussi envisager un approvisionnement en partie via des circuits commerciaux, supposant une récolte à plus longue distance du site dans des zones plus en altitude, mais il ne faut pas négliger l'hypothèse de la survie à basse altitude de peuplements épars de ces espèces dans des zones protégées, pour expliquer la présence des taxons collinéens et montagnards (Durand, 1998; Chabal, 1997; Puertas, 1998; Delhon et Thiébault, 2005). Le Paillon traverse l'arrière-pays dans une vallée assez étroite alimentée par trois rivières et quelques ruisseaux issus des premiers reliefs alpins ayant leurs sources vers $1300 \mathrm{~m}$ d'altitude, près du Col de l'Ablé (NicolPichard, 1982). On peut aussi formuler l'hypothèse de la récolte de bois flotté, venu des hauts sommets et récupérés le long des rives du Paillon, après des crues ou sur la grève après une tempête, ce qui soulignerait le caractère opportuniste du ramassage des bois et expliquerait la présence de taxons montagnards (Le Lay et Piégay, 2007). Cette pratique de ramassage de bois flotté pour une utilisation comme combustible domestique est documentée par les textes en Camargue au haut Moyen Âge (Durand et Ruas, 2004).

L'utilisation de combustible issu de la coupe de bois «sur pied ", via des circuits d'approvisionnement depuis des zones dédiées à la production de bois n'est pas démontrable à partir de nos résultats, qui sont tout à fait compatibles avec une récolte opportuniste se portant en très grande majorité sur la biomasse présente à proximité immédiate du site.

La biodiversité relativement importante des spectres est en faveur de l'utilisation, comme combustible, de bois plutôt que de charbon de bois produit par charbonnage. En effet, la production de charbon de bois se fait plutôt à partir d'essences et de parcelles sélectionnées et aboutit à des spectres de faible biodiversité (Fabre et al., 1990). En revanche, il n'est pas possible, en l'état actuel des connaissances, de différencier l'utilisation de bois de l'utilisation de charbon de bois comme combustible à partir de l'observation microscopique des charbons (Ludemann et Nelle, 2002; Ludemann, 2006).

Les données recueillies peuvent être rapprochées de celles issues de l'atelier de fonderie du site marseillais du Xvi ${ }^{\mathrm{e}}$ $\mathrm{XVII}^{\mathrm{e}}$ siècle pour ce qui concerne la végétation (Mellinand et Thomas, 2011). En effet, les principaux taxons utilisés sur le site marseillais sont semblables (chênes, olivier, pins, genévrier, érable, arbousier, noyer, bruyère, vigne, alaterne/ filaire, pistachier) à ceux identifiés sur le site de la colline du Château de Nice. À l'heure actuelle, nous n'avons pas pu effectuer de mesure des calibres des bois utilisés comme combustible à Nice pour compléter l'analyse anthracologique. Ces données nous renseigneraient sur la gestion de la ressource en bois (Figueiral-Rowe, 2011; Paradis-Grenouiller et al., 2010; Chrzavzez et al., 2011). En effet, I. FigueiralRowe (2011) suggère, non seulement, que la diversité taxinomique témoignerait d'un comportement non-sélectif vis-à-vis des essences méditerranéennes typiques, provenant des alentours de la ville avec un apport plus lointain, probablement par flottage, mais que l'utilisation de bois de petits calibres pourrait être liée aux contraintes de la combustion. 


\section{Activités artisanales}

Les charbons de bois des US 2291 et 2297 situées au fond de la fosse FS 388 peuvent être envisagés comme des rejets primaires, c'est-à-dire déposés directement depuis l'activité qui les a produits et retrouvés au même emplacement. En effet, les fragments de charbons de bois sont presque exclusivement issus du chêne à feuillage caduc, du chêne vert et de l'arbousier, et ils proposent donc un spectre floristique très caractéristique. Ces US ne présentent pas ou peu de contamination par du mobilier ou des ossements (Bouiron, 2011). La présence d'une bûchette en place dans l'US 2297 indique le caractère instantané d'une partie du dépôt. Cela confirme l'hypothèse de l'utilisation de la fosse uniquement pour le feu de la coulée de la cloche. Il est intéressant de souligner que, outre le paramètre important du calibre, la densité du bois conditionnerait les propriétés du combustible (Thery-Parisot, 2001; Thomas 2009b). Les essences dont le bois a une faible densité (saule, peuplier, aulne) sont préférables pour initier une combustion car elles favorisent la production de flammes plutôt que de braises. Au contraire, les essences plus denses (chêne, charme, orme), parce que moins poreuse, entretiennent plus longtemps la comburation (oxydation plus lente) et conviennent par exemple pour chauffer durablement un lieu clos (Chabal et al., 1999). Il est actuellement impossible de généraliser les résultats et cette première étude sera complétée. Le choix des essences est davantage une possibilité qu'une nécessité (Théry-Parisot et al., 2010) sans exclure d'autres possibilités.

Concernant la fosse FS 321 et les cinq trous de poteaux analysés, les charbons de bois semblent être des rejets secondaires, ce qui signifie que les fragments ont été déplacés au moins une fois, volontairement ou non, à distance de leur lieu de production. En effet, non seulement les essences sont variées (table 1), mais de plus le mobilier est très diversifié et les ossements en nombre important (Bouiron, 2011). Le contenu du comblement suggère une utilisation des structures en creux comme dépotoir. La fonction du bois retrouvé sous forme carbonisée ne nous est pas connue, mais il complète la liste floristique obtenue à partir des fosses des moules à cloche, en particulier en ce qui concerne la ripisylve, bien représentée, que l'on situe volontiers sur les rives du Paillon, au pied de la colline du Château ou plus en amont.

Le fonctionnement technique des fours pour la coulée des cloches et pour la fonte du métal en général fait l'objet d'études récentes (Thomas, 2009a; Thomas, 2010). D'autres études, aussi bien anthracologiques que textuelles, concernent les fours à chaux en Provence au Moyen Âge (Vaschalde, 2013) ou les fours de potiers gallo-romains (Chabal et al., 1999). Ces études montrent qu'un large cor- tège d'essences est utilisé, sans qu'aucune explication technique ne puisse justifier le choix du combustible. Dans les fosses, nous avons retrouvé des morceaux de bois imprégnés d'un alliage cuivreux, qu'il serait nécessaire d'analyser pour connaitre sa composition et définir l'étape concernée (affinage, fusion du cuivre, addition d'étain). Nous poursuivrons cette étude afin de préciser les pratiques artisanales. En effet, il serait très intéressant d'avoir accès à l'ensemble des opérations comme cela a été décrit pour un atelier de cloche dans la Villeneuve du Temple à Paris (Thomas, 2009a). Toutefois, nous ne disposons que des vestiges du four de cuisson $\mathrm{du}$ moule à cloche et non pas du four de fusion du métal, sauf si l'on considère que lorsque ce dernier a été détruit, ses restes ont pu être vidangés dans les fosses de coulée de cloche. Il ne nous est actuellement pas possible de comparer le combustible provenant des deux structures.

Par ailleurs, l'approvisionnement en métal pour couler une cloche de taille moyenne, dont le diamètre est estimé à 60-70 cm dans la fosse FS 388 et à $70-75 \mathrm{~cm}$ pour la fosse FS 321 (Damotte, 2011), est une question qu'il est nécessaire de poser mais qui reste difficile à approcher lorsque les cloches n'existent plus. On peut souligner qu'aucune cloche médiévale n'est connue à Nice; la plus ancienne présente dans la Tour Rusca, place du palais de justice, date des années 1666 (Baréty, 1913).

\section{Conclusion}

Les essences utilisées comme combustible dans les fosses de coulée de cloche sur le site de la colline du château sont pour la fosse FS 388 majoritairement le chêne et l'arbousier, alors que le taxon peuplier/saule domine dans la fosse FS 321. L'ensemble des données suggère que les formations ligneuses fréquentées par les habitants de la colline du château pour l'approvisionnement en combustible sont surtout mésoméditerranéennes et supraméditerranéennes d'adret et pourraient également appartenir à des boisements de la transition entre les étages supraméditerranéen d'ubac et montagnard. Il y a donc une cohérence entre la situation géographique du site (100 m d'altitude, exposition sud et enserré dans un cirque de collines et de montagnes qui voit la transition des étages mésoméditeranéen et supramediterranéen localisée vers $1000 \mathrm{~m}$ d'altitude) et la répartition biogéographique potentielle des taxons déterminés. Nous ne disposons pas en l'état actuel de la documentation d'information sur la localisation plus précise de l'aire d'approvisionnement en combustible, ni sur son étendue, ni sur sa morphologie, ou la composition totale des formations ligneuses exploitées. 
Ces premières données limitées à un seul site archéologique seront complétées par des analyses en cours sur d'autres sites du Sud de la France. Ainsi, nous aurons accès à tout un pan méconnu de la chaîne opératoire de la fabrication des cloches qui nous permettra, in fine, de mieux connaître les techniques des saintiers, leurs circuits d'approvisionnement en combustible et leur place au sein du tissu socio-économique.

\section{Remerciements}

Cette étude a été possible grâce au Programme Collectif de Recherche dirigé par Marc Bouiron et le Service Archéologie de la ville de Nice/CEPAM, dont nous tenons à remercier tous les collaborateurs. Cette étude a été faite en partie dans le cadre du Master Histoire et Archéologie des Mondes Anciens et Médiévaux de l'Université Nice Sophia Antipois dirigé par le Professeur Michel Lawers, sous la direction de Yann Codou et Claire Delhon.

\section{Bibliographie}

Bats J.-C., 2010. Pendelle, Escaudes (33) : Entre Landes et Ciron, une occupation médiévale et son four à cloche, Rapport final d'opération de fouilles archéologiques, Institut national de recherches archéologiques préventives, Service Régional de l'Archéologie Aquitaine, Bordeaux.

Baréty A., 1913. Nice Historique, enquête campanaire. 39, 55-59. Bernazzani C., 2009. Le firme dei magistri campanarum nel Medioevo. Un'indagine fra Parma et Piacenza. Opera-NominaHistoriae. 1 : 99-136.

Bouiron M. (coord.) 2011. Programme Collectif de RechercheLa colline du Château à Nice - Rapport triennal 2009-2011. Ville de Nice-CEPAM-SRA.

Bouiron M. (dir.), 2013. Nice. La colline du Château. Histoire millénaire d'une place forte. Nice, Mémoires millénaires, 303 p.

Bouiron M., Mercurin R., 2007. Les fouilles anciennes. In M. Bouiron (dir.), Projet Collectif de Recherche, La colline du Château à Nice, l'occupation des origines à nos jours, Ville de Nice, CEPAM-SRA, 217-520.

Chabal L., 1994. Apports récents de l'anthracologie à la connaissance des paysages passés : performance et limites. Histoire et mesure, IX-3/4 : 317-338.

Chabal L., 1997. Forêts et sociétés en Languedoc (Néolithique final, Antiquité tardive). L'anthracologie, méthode et paléoécologie, Documents Archéologie Française, 63, Paris.
Chabal L., Fabre L., Terral J.-F., Théry-Parisot I., 1999. L'Anthracologie, in A. Ferdière (dir.), La Botanique, Paris, Errance, coll. "Archéologiques », 43-104.

Chrzavzez J., Henry A., Théry-Parisot I., 2011. Identificando estrategias de adquisición del combustible leñoso en antracología: ¿puede contribuir la experimentación a determinar el calibre de los carbones en contexto arqueológico? La Investigación Experimental aplicada en la Arqueología. Antonio Morgado Javier Baena Preysler David García Gonzalez (eds). Departamento de Prehistoria y Arqueología de la Universidad de Granada. Bloque II. Capitulo XXIV. 205-211.

Damotтe L. 2011. Les fosses de coulées de cloches. In M. Bouiron (dir.), Projet Collectif de Recherche, La colline du Château à Nice, l'occupation des origines à nos jours, Ville de Nice, CEPAMSRA, 88-99.

Delhon C., 2006. Paleoecological reliability of pedo-anthracological assemblages. In A. Dufraisse (ed.), Charcoal Analysis: New Analytical Tools and Methods for Archaeology: Papers from the Table-Ronde Held in Basel 2004. Archaeopress, Oxford, 9-24.

Delhon C., Thiebault S., 2005. The migration of beech (Fagus Salvatica L.) up the Rhone: the mediterranean history of a "mountain" species. Journal of Vegetation history and Archaeobotany 14, 119-132.

Durand A., 1998. Les paysages médiévaux du Languedoc ( $\mathrm{x}^{\mathrm{e}}$ $\mathrm{XII}^{\mathrm{e}}$ siècles), Toulouse, Presses universitaires du Mirail.

Durand A., Ruas M.-P., 2004. La forêt languedocienne IX ${ }^{\mathrm{e}}$ $\mathrm{XI}^{\mathrm{e}}$ siècle. In A. Corvol, D'Occident du Moyen Âge à nos jours, Actes des $24^{\mathrm{e}}$ journées internationales de Flaran (Gers, septembre 2002), Toulouse, Presses universitaires du Mirail, p. 163-180 b (Flaran, 24).

Fabre L., Grau Almero E., Lalanne J.-F., Vernet J.-L., Durand A., 1990. Charbonnières et forêt méditerranéenne à la Boissière (Hérault). In J.-P. Métaillié (dir.), Protoindustries et histoire des forêts, Actes du colloque tenu à Loubières (Ariège) du 10 au 13 octobre 1990, Toulouse, GDR Isard, CNRS, Université de Toulouse le Mirail, 1992, p. 287-300, Les Cahiers de l'Isard, p. 237-255.

Figueiral-Rowe I., 2011. Le combustible. In P. Mellinand, N. Thomas (dir.), 2011 L'Hôtel-Dieu, Marseille, Rapport final d'opération de fouilles archéologiques, Institut national de recherches archéologiques préventives, Service Régional de l'Archéologie des Bouches-du-Rhône, Marseille, 193-205.

Gonon T., 2000. Les cloches du sud-est de la France : fabrication et évolution typologique au cours du Moyen Âge. In P. Petrequin, P. Fluzin, J. Thiriot, P. Benoit, Arts du feu et productions artisanales. XX ${ }^{\mathrm{e}}$ Rencontres Internationales d'Archéologie et d'Histoire d'Antibes, Éd. APDCA, Antibes, 205-220.

Gonon T., 2002. Les cloches en France au Moyen Âge : Étude archéologique et approche Historique, thèse, université de Lyon II. 
Gonon T., 2010. Les cloches en France au Moyen Âge. Archéologie d'un instrument singulier. Paris, Errance.

Le Lay Y.-F., Piegay H., 2007. Le bois mort dans les paysages fluviaux français : éléments pour une gestion renouvelée, L'Espace géographique, 36 : 51-64.

Ludemann T., Nelle O., 2002. Die Wälder am Schauinsland und ihre Nutzung durch Bergbau und Köhlerei. Freiburg, Forstwissenschatliche Fakultät der Universität Freiburg und Forstlische Versuchs- und Forshungsanstalt BadenWürttemberg.

Ludemann T., 2006. Anthracological analysis of recent charcoalburning in the Black Forest, SW Germany. In A. Dufraisse (ed.), Charcoal Analysis: New Analytical Tools and Methods for Archaeology: Papers from the Table-Ronde Held in Basel 2004. Archaeopress, Oxford, 61-70.

Mellinand P., Thomas N. (dir.), 2011. L'Hôtel-Dieu, Marseille, Rapport final d'opération de fouilles archéologiques. Institut national de recherches archéologiques préventives, Service Régional de l'Archéologie des Bouches-du-Rhône, Marseille, 2 volumes, $517 \mathrm{p}$.

Nicol-Pichard S., 1982 Analyse pollinique de sédiments littoraux postglaciaires de l'embouchure du Paillon (Nice). Ecologia Mediterranea, Tome $8: 87-95$.

Ozenda P., 1956. Carte de la végétation de la France à 1/200 000, feuille de Nice, $\mathrm{n}^{\circ}$ 68, CNRS Éditions.

Ozenda P., 1975. Sur les étages de végétation dans les montagnes du bassin méditerranéen. Doc. Cartographie Ecol., 16 : 1-32.

Paradis-Grenouillet S., Leleu J.-P., Belingard C., Rouaud R., AlléE P., 2010, AnthracoLoJ, un outil pour la simplification des mesures dendrométriques. Panorama de la dendrochrologie en France 8-10 octobre 2009, Digne, p. 197-202. Cahiers de Géographie, EDYTEM, vol n 11.

Pons A., Quézel P., 1998. À propos de la mise en place du climat méditerraneen. C.R.Acad. Sci. Paris, Sciences de la terre et des planètes. 327, 755-760.

Puertas O., 1998. Palynologie dans le delta du Lez : contribution à l'histoire du paysage de Lattes, ARALO Lattes, Lattara, 11-181.

Quezel P., 1976. Les chênes sclérophylles en région méditerranéenne, CIHEAM, options méditerranéennes, 35 : 25-29.

Rameau J.-C., Mansion, D., Dume, G., Gauberville, C., 2008. Flore Forestière française. Guide écologique illustré. Volume 3. Institut pour le développement forestier-CNPPF.
Schweingruber F. H., 1978. Anatomie microscopique du bois. Edition Zürcher AG, Suisse.

Sivan O., Court-Picon M., 2007. Évolution géomorphologique holocène des plaines alluviales niçoises. Archéam, 14 : 1-17.

Thery-Parisot I., 2001. L'économie des combustibles au Paléolithiques. CEPAM, Dossiers de documentation archéologique. $\mathrm{N}^{\circ}$ 20, CNRS Éditions, Paris 195 p.

Théry-Parisot I., Chabal L., Ntinou M., Bouby L., Carré A., 2010. Du bois aux charbons de bois : approche expérimentale de la combustion. P@lethnologie, 2 : 81-93.

Thévenon U., 1971. Campagne de fouilles 1969-1970 sur le site de Vagnas (Ardèche). Revue archéologique du Centre de la France. 10 (3): 328-328.

Thomas N., 2009a. L'atelier de fonte de cloches. In M. Goy (dir.), Fouille au 4, rue des Visitandines dans l'enceinte castrale à Montbrison (42), Rapport final d'opération de fouilles archéologiques, Institut national de recherches archéologiques préventives, Service Régional de l'Archéologie Rhône-Alpes, Lyon, 82-109.

Thomas N., 2009b. Les ateliers urbains de travail du cuivre et de ses alliages au bas Moyen Âge: Archéologie et histoire d'un site parisien du xive siècle dans la Villeneuve du Temple (1325-1350), Thèse de l'Université Paris 1 Panthéon-Sorbonne, France.

Thomas N., 2010. Fonte d'une cloche à la fin du XIve ou au début du $\mathrm{xv}^{\mathrm{e}}$ siècle. In J.-C. Bats (dir.), Pendelle, Escaudes (33): Entre Landes et Ciron, une occupation médiévale et son four à cloche, Rapport final d'opération de fouilles archéologiques, Institut national de recherches archéologiques préventives, Service Régional de l'Archéologie Aquitaine, Bordeaux, 2010, p. 68-111.

Vaschalde C., 2013. L'art de cuire la pierre en France méditerranéenne à la fin du Moyen Âge. Approche interdisciplinaire d'un artisanat méconnu : la chaufournerie, thèse de l'université de Provence (Durand A., Thiriot J. dir.), 3 vol., 1004-1478 p.

Vernet J.-L., Ogereau P., Figueiral I., Machado Yanes C., Uzquiano P., 2001. Guide d'identification des charbons de bois préhistorique et récents. Sud-Ouest de l'Europe : France, péninsule ibérique et îles Canaries. CNRS Éditions. 